\title{
Composition of happy functions
}

\author{
Passawan Noppakeaw $^{1}$, Niphawan Phoopha ${ }^{2}$ \\ and Prapanpong Pongsriiam ${ }^{3, *}$ \\ ${ }^{1}$ Department of Mathematics, Faculty of Science \\ Silpakorn University, Nakhon Pathom, 73000, Thailand \\ e-mail: p.noppakeaw@gmail.com, noppakeaw_p@silpakorn.edu \\ ${ }^{2}$ Department of Mathematics, Faculty of Science \\ Silpakorn University, Nakhon Pathom, 73000, Thailand \\ e-mail: phoopha.miw@gmail.com, phoopha_n@silpakorn.edu \\ ${ }^{3}$ Department of Mathematics, Faculty of Science \\ Silpakorn University, Nakhon Pathom, 73000, Thailand \\ e-mail: prapanpong@gmail.com, pongsriiam_p@silpakorn.edu
}

* Corresponding author.

Received: 28 August 2018

Abstract: For positive integers $e \geq 1$ and $b \geq 2$, let $S_{e, b}: \mathbb{N} \rightarrow \mathbb{N}$ be defined by

$$
S_{e, b}(x)=a_{k}^{e}+a_{k-1}^{e}+\cdots+a_{1}^{e}
$$

if $x=\left(a_{k} a_{k-1} \cdot a_{1}\right)_{b}=a_{k} b^{k-1}+a_{k-1} b^{k-2}+\cdots+a_{2} b+a_{1}$ is the expansion of $x$ in base $b$. We call $S_{e, b}$ an $(e, b)$-happy function. Let $g$ be a composition of various $(e, b)$-happy functions. We show that, for any given $x \in \mathbb{N}$, the iteration sequence $\left(g^{(n)}(x)\right)_{n \geq 0}$ either converges to a fixed point or eventually becomes a cycle. Here $g^{(0)}$ is the identity function mapping $x$ to $x$ for all $x$ and $g^{(n)}$ is the $n$-fold composition of $g$. In addition, we prove that the number of all possible fixed points and cycles is finite. Examples are also given.

Keywords: Happy number, Happy function, Digit, Dynamic, Iteration.

2010 Mathematics Subject Classification: 11A63, 26A18. 


\section{Introduction and main results}

Let $S_{2}: \mathbb{N} \rightarrow \mathbb{N}$ be the function that takes a positive integer to the sum of the squares of its decimal digits. Then, $S_{2}$ is called the happy function and if $S_{2}^{(n)}(x)=1$ for some $n \geq 1$, Then, $x$ is called a happy number (see [1] and [5, Chapter E34]). For $n \geq 1$ and a function $f: \mathbb{N} \rightarrow \mathbb{N}, f^{(0)}$ is the identity function and $f^{(n)}$ is the $n$-fold composition of $f$. For example, the sequence $\left(S_{2}^{(n)}(7)\right)_{n \geq 0}$ is $(7,49,97,130,10,1,1, \ldots)$ which converges to the fixed point 1 and the sequence $\left(S_{2}^{(n)}(2)\right)_{n \geq 0}$ is $(2,4,16,37,58,89,145,42,20,4, \ldots)$ which is eventually the cycle $(4,16, \ldots, 20)$. It is well-known (see [1] or [5]) that for any $x \in \mathbb{N},\left(S_{2}^{(n)}(x)\right)_{n \geq 0}$ either converges to 1 or becomes the cycle $(4,16, \ldots, 20)$. As usual, $\left(a_{1}, a_{2}, \ldots, a_{k}\right)$ and any cyclic permutation such as $\left(a_{k-1}, a_{k}, a_{1}, a_{2}, \ldots, a_{k-2}\right)$ are considered the same cycle. By the above, we see that 7 is happy but 2 is not. See also Sequence A007770 in OEIS [8] for a list of happy numbers and other information. More generally, for positive integers $e \geq 1$ and $b \geq 2$, we define $S_{e, b}: \mathbb{N} \rightarrow \mathbb{N}$ by

$$
S_{e, b}(x)=a_{k}^{e}+a_{k-1}^{e}+\cdots+a_{1}^{e},
$$

if $x=\left(a_{k} a_{k-1} \ldots a_{1}\right)_{b}=a_{k} b^{k-1}+a_{k-1} b^{k-2}+\cdots+a_{2} b+a_{1}$ is the expansion of $x$ in base $b$. We call $S_{e, b}$ an $(e, b)$-happy function and if there exists $n \geq 1$ such that $S_{e, b}^{(n)}(x)=1$, then $x$ is said to be an $(e, b)$-happy number (see [3] and [6]). For convenience, if we write a number without specifying a base, it is always written in base 10. Grundman and Teeple [4] obtain a result which implies that if $x, e, b$ are given, then the sequence

$$
\left(S_{e, b}^{(n)}(x)\right)_{n \geq 0} \text { converges to a fixed point or eventually becomes a cycle. }
$$

In this article, we generalize (1) and (2) to the following form.

Definition 1.1. For each $\underline{\boldsymbol{e}}=\left(e_{1}, \ldots, e_{k}\right)$ and $\underline{\boldsymbol{b}}=\left(b_{1}, \ldots, b_{k}\right)$ with $e_{i} \geq 1$ and $b_{i} \geq 2$ for all $i=1,2, \ldots, k$, define $S_{\underline{e}, \underline{b}}: \mathbb{N} \rightarrow \mathbb{N}$ by

$$
S_{\underline{\boldsymbol{e}}, \underline{\boldsymbol{b}}}(x)=\left(S_{e_{1}, b_{1}} \circ S_{e_{2}, b_{2}} \circ \cdots \circ S_{e_{k}, b_{k}}\right)(x) \quad \text { for all } x \in \mathbb{N} .
$$

If $x \in \mathbb{N}$ and $S_{\underline{\boldsymbol{e}}, \underline{\boldsymbol{b}}}^{(n)}(x)=1$ for some $n \geq 1$, then $x$ is said to be $(\underline{\boldsymbol{e}}, \underline{\boldsymbol{b}})$-happy.

So if $e_{1}=e_{2}=\cdots=e_{k}=e$ and $b_{1}=b_{2}=\cdots=b_{k}=b$, then the iteration sequence $\left(S_{\underline{\boldsymbol{e}}, \underline{\underline{b}}}^{(n)}(x)\right)_{n \geq 0}$ is a subsequence of $\left(S_{e, b}^{(n)}(x)\right)_{n \geq 0}$ but if $e_{i}$ or $b_{i}$ are not all equal, then $\left(S_{\underline{\boldsymbol{e}}, \underline{\boldsymbol{b}}}^{(n)}(x)\right)_{n \geq 0}$ may be a totally different sequence. For instance, suppose $\underline{\boldsymbol{e}}=(3,2), \underline{\boldsymbol{b}}=(4,10)$, and $x=7$. Then, $S_{\underline{e}, \underline{b}}(x)=\left(S_{3,4} \circ S_{2,10}\right)(7)=S_{3,4}\left(S_{2,10}(7)\right)=S_{3,4}\left(7^{2}\right)=S_{3,4}(49)=S_{3,4}\left((301)_{4}\right)=$ $3^{3}+0^{3}+1^{3}=28$. $S_{\underline{e}, \underline{b}}(28)=\left(S_{3,4} \circ S_{2,10}\right)(28)=S_{3,4}\left(2^{2}+8^{2}\right)=S_{3,4}(68)=S_{3,4}\left((1010)_{4}\right)=$ $1^{3}+0^{3}+1^{3}+0^{3}=2$. So the sequence $\left(S_{\underline{e}, \underline{b}}^{(n)}(7)\right)_{n \geq 0}$ is $(7,28,2,1,1, \ldots)$, and so 7 and 2 are $(\underline{\boldsymbol{e}}, \underline{\boldsymbol{b}})$-happy numbers. Our purpose is to show that (2) also holds when $S_{e, b}$ is replaced by $S_{\underline{e}, \underline{b}}$. The proof can be obtained from a general method as follows. For a function $f: \mathbb{N} \rightarrow \mathbb{N}$, define the following two conditions:

(A) There exists $N_{f} \in \mathbb{N}$ such that $f(x)<x$ for all $x \geq N_{f}$.

(B) For each $x \in \mathbb{N}$, the sequence $\left(f^{(n)}(x)\right)_{n \geq 0}$ converges to a fixed point of $f$ or eventually enters into a cycle. In addition, the number of all such fixed points and cycles is finite. 
Then, the generalization of (2) to $S_{\underline{e}, \underline{b}}$ follows from the following two theorems.

Theorem 1.2. If $f: \mathbb{N} \rightarrow \mathbb{N}$ satisfies (A), then $f$ satisfies $(B)$.

Theorem 1.3. If $f_{1}, f_{2}, \ldots, f_{k}: \mathbb{N} \rightarrow \mathbb{N}$ satisfy $(A)$, then their composition $f_{1} \circ f_{2} \circ \cdots \circ f_{k}$ also satisfies (A).

Remark that the idea of Theorem 1.2 is not new; for example, it is used in the proof of the main result in [4] in the case $f=S_{e, b}$. Nevertheless, Theorem 1.2 for a general function $f$ seems to be new, and as far as we are aware Theorem 1.3 is also new and it leads us to the following theorem.

Theorem 1.4. The function $S_{\underline{e}, \underline{b}}$ defined by (3) satisfies $(A)$ and (B).

For more details about happy numbers and happy functions, see for example [2,7,9-11] and the references therein.

\section{Proof of the main results}

Proof of Theorem 1.2. For convenience, we write $N$ instead of $N_{f}$ and we assert that

$$
\text { for every } y \in \mathbb{N} \text {, there exists } n \in \mathbb{N} \text { such that } f^{(n)}(y)<N \text {. }
$$

Suppose that there exists $y \in \mathbb{N}$ such that $f^{(n)}(y) \geq N$ for every $n \in \mathbb{N}$. Since $f(y) \geq N$, we obtain by (A) that $f(f(y))<f(y)$. Since $f^{(2)}(y) \geq N$, we apply (A) again and obtain $f^{(3)}(y)<f^{(2)}(y)<f(y)$. Let $k \in \mathbb{N}$ be any positive integer. Since $f^{(n)}(y) \geq N$ for every $n \in \mathbb{N}$, we can repeat the above argument $k$ times and obtain a strictly decreasing sequence of positive integers $f(y)>f^{(2)}(y)>f^{(3)}(y)>\cdots>f^{(k)}(y)$. Since these are integers, we have

$$
f(y) \geq f^{(2)}(y)+1 \geq f^{(3)}(y)+2 \geq \cdots \geq f^{(k)}(y)+k-1 .
$$

Since (5) holds for any $k$, we can choose $k=f(y)+1$, and obtain $f^{(k)}(y) \leq f(y)-(k-1)=0$, which is a contradiction. Hence, (4) is proved.

Now let $x \in \mathbb{N}$ and suppose that $\left(f^{(n)}(x)\right)_{n \geq 0}$ does not converge to a fixed point of $f$. By (4), there exists $n_{1} \in \mathbb{N}$ such that $f^{\left(n_{1}\right)}(x)<N$. Again by (4), there exists $n_{2} \in \mathbb{N}$ such that $f^{\left(n_{2}\right)}\left(f^{\left(n_{1}\right)}(x)\right)<N$. Repeating this process $N$ times, we obtain the set of positive integers

$$
f^{\left(n_{1}\right)}(x), f^{\left(n_{1}+n_{2}\right)}(x), \ldots, f^{\left(n_{1}+n_{2}+\cdots+n_{N}\right)}(x)
$$

which are less than $N$. By the pigeonhole principle, some of them are the same, say

$$
f^{\left(n_{1}+n_{2}+\cdots+n_{j}\right)}(x)=f^{\left(n_{1}+n_{2}+\cdots+n_{j}+\cdots+n_{\ell}\right)}(x) \quad \text { for some } \ell>j \geq 1 .
$$

Let $y=f^{\left(n_{1}+n_{2}+\cdots+n_{j}\right)}(x)$. Then, the tail of the sequence $\left(f^{(n)}(x)\right)_{n \geq 0}$ eventually becomes

$$
\left(y, f(y), f^{(2)}(y), \ldots, f^{\left(n_{j+1}+n_{j+2}+\cdots+n_{\ell}-1\right)}(y), y, \ldots\right)
$$


which is a cycle. This proves the first part of (B). Next, we show that the set $U_{f}$ of fixed points and cycles is finite. More precisely, we will show that

$$
U_{f}:=\left\{x \in \mathbb{N} \mid \exists n \in \mathbb{N}, f^{(n)}(x)=x\right\} \subseteq[1, M],
$$

where $M=\max \{N, f(1), f(2), \ldots, f(N)\}$. First of all, by (A), if $x$ is a fixed point of $f$, then $x<N$ and so $x \in[1, M]$. Suppose that $x$ is an element in a cycle arising from the iteration $\left(f^{(n)}(y)\right)_{n \geq 0}$ for some $y \in \mathbb{N}$. If $x<N$, then $x \in[1, M]$ and we are done. So suppose $x \geq N$. By (4), there exists $n \in \mathbb{N}$ such that $f^{(n)}(x)<N$. Since $x$ is in a cycle, after some iterations, it must come back to $x$. That is, there exists $k \in \mathbb{N}$ such that $f^{(k)}\left(f^{(n)}(x)\right)=x$. If $k=1$ or $f^{(n+k-1)}(x) \leq N$, then $x=f\left(f^{(n+k-1)}(x)\right) \leq M$ and we are done. So suppose $k \geq 2$ and $f^{(n+k-1)}(x)>N$. Let $\ell$ be the smallest positive integer such that $f^{(n+k-\ell)}(x)<N$. Then, $\ell>1$ and for each $1 \leq i<\ell, f^{(n+k-i)}(x) \geq N$. So

$$
f^{(n+k-\ell+1)}(x)>f^{(n+k-\ell+2)}(x)>\cdots>f^{(n+k-1)}(x)>f^{(n+k)}(x)=x .
$$

So $x<f^{(n+k-\ell+1)}(x)=f\left(f^{(n+k-\ell)}(x)\right) \leq M$. Therefore, (6) is verified and the proof is complete.

Proof of Theorem 1.3. We prove this by induction on $k$. When $k=1$, the result is obvious. Assume that $k \in \mathbb{N}$ and the result holds for $k$. Suppose that $f_{1}, f_{2}, \ldots, f_{k+1}: \mathbb{N} \rightarrow \mathbb{N}$ satisfy (A). Let $f=f_{1} \circ f_{2} \circ \cdots \circ f_{k+1}$ and $g=f_{1} \circ f_{2} \circ \cdots \circ f_{k}$. Then, there are $m_{1}, m_{2} \in \mathbb{N}$ such that

$$
g(x)<x \quad \text { for all } x \geq m_{1}, \text { and } f_{k+1}(x)<x \quad \text { for all } x \geq m_{2} .
$$

Let $m_{3}=\max \left\{g(x) \mid 1 \leq x<m_{1}\right\}$ and $m=\max \left\{m_{1}, m_{2}, m_{3}\right\}+1$. Let $x \geq m$. We will show that $f(x)<x$. If $f_{k+1}(x) \geq m_{1}$, then we obtain by (7) that

$$
f(x)=g\left(f_{k+1}(x)\right)<f_{k+1}(x)<x .
$$

On the other hand, if $f_{k+1}(x)<m_{1}$, then $f(x)=g\left(f_{k+1}(x)\right) \leq m_{3}<m \leq x$. This completes the proof.

Proof of Theorem 1.4. Grundman and Teeple [4, Theorem 1] show that if $x \geq b^{e+1}$, then $S_{e, b}(x)<x$. That is, $S_{e, b}$ has property (A) for every $e \geq 1$ and $b \geq 2$. By Theorem 1.3, $S_{\underline{e}, \underline{b}}$ also satisfies (A). Then, by Theorem 1.2, we obtain that $S_{\underline{e}, \underline{b}}$ satisfies (B), as desired.

We remind the reader again that if we write a number without specifying a base, it is always written in base 10. We show some explicit calculations in the following examples.

Suppose $\underline{\boldsymbol{e}}=\left(e_{1}, e_{2}, \ldots, e_{k}\right), \underline{\boldsymbol{b}}=\left(b_{1}, b_{2}, \ldots, b_{k}\right)$, and $f=S_{\underline{e}, \boldsymbol{b}}$. By Theorem $1.4, f$ satisfies (A), that is, there exists $N \in \mathbb{N}$ such that $f(x)<x$ for all $x \geq N$. We can find such $N$ by the argument given in the proof of Theorems 1.2, 1.3, and 1.4.

Example 2.1. Consider $f=S_{4,6} \circ S_{2,5} \circ S_{3,4} \circ S_{5,3}$, which is the last line of Table 1. By the proof of Theorem 1.4, we know that

$$
S_{4,6}(x)<x \quad \text { for } x \geq 6^{5} \quad \text { and } \quad S_{2,5}(x)<x \quad \text { for } x \geq 5^{3} .
$$


In the proof of Theorem 1.3, we let $m_{1}=6^{5}, m_{2}=5^{3}, m_{3}=\max \left\{S_{4,6}(x) \mid 1 \leq x<6^{5}\right\}=5^{5}$, and $m=\max \left\{m_{1}, m_{2}, m_{3}\right\}+1=6^{5}+1$. Then

$$
\left(S_{4,6} \circ S_{2,5}\right)(x)<x \quad \text { for } x \geq 6^{5}+1 .
$$

Again by the proof of Theorem 1.4, we have $S_{3,4}(x)<x$ for $x \geq 4^{4}$. By the proof of Theorem 1.3, we let $m_{1}=6^{5}+1, m_{2}=4^{4}$,

$$
\begin{aligned}
m_{3} & =\max \left\{\left(S_{4,6} \circ S_{2,5}\right)(x) \mid 1 \leq x<6^{5}+1\right\} \\
& \leq \max \left\{S_{4,6}(x) \mid 1 \leq x \leq 96\right\} \\
& <S_{4,6}\left((255)_{6}\right)=1266,
\end{aligned}
$$

and $m=\max \left\{m_{1}, m_{2}, m_{3}\right\}+1=6^{5}+2$. Then, $\left(S_{4,6} \circ S_{2,5} \circ S_{3,4}\right)(x)<x$ for $x \geq 6^{5}+2$. Finally, we know that $S_{5,3}(x)<x$ for $x \geq 3^{6}$, so we let $m_{1}=6^{5}+2, m_{2}=3^{6}$,

$$
\begin{aligned}
m_{3} & =\max \left\{\left(S_{4,6} \circ S_{2,5} \circ S_{3,4}\right)(x) \mid 1 \leq x<6^{5}+2\right\} \\
& \leq \max \left\{\left(S_{4,6} \circ S_{2,5}\right)(x) \mid 1 \leq x \leq 189\right\} \\
& \leq \max \left\{\left(S_{4,6}(x)\right) \mid 1 \leq x \leq 49\right\} \\
& \leq S_{4,6}\left((155)_{6}\right)=1251,
\end{aligned}
$$

and $m=\max \left\{m_{1}, m_{2}, m_{3}\right\}+1=6^{5}+3$. Hence

$$
f(x)=\left(S_{4,6} \circ S_{2,5} \circ S_{3,4} \circ S_{5,3}\right)(x)<x \quad \text { for all } x \geq 6^{5}+3 .
$$

This shows an algorithm to obtain an $N$ satisfying the condition (A). This choice of $N$ may not be optimal but if it is necessary, we can find the minimal $N$ by checking if $f(x)<x$ for $x=N-1, N-2, N-3, \ldots$ and then we stop when we get the first $x$ such that $f(x) \geq x$. Then, we use a computer to find all fixed points and cycles of $f$ by checking the sequence $\left(f^{(n)}(x)\right)_{n \geq 0}$ where $x=1,2,3, \ldots, N$.

We give two more examples to illustrate alternative calculations.

Example 2.2. Let $\underline{\boldsymbol{e}}=(3,2), \underline{\boldsymbol{b}}=(10,10)$, and $f=S_{\underline{\boldsymbol{e}}, \underline{\boldsymbol{b}}}$. That is, $f=S_{3,10} \circ S_{2,10}$. Then, for each $x \in \mathbb{N}$, the sequence $\left(f^{(n)}(x)\right)_{n \geq 0}$ contains either 1 or 27 . Moreover, 1 is the only fixed point of $f$ and if the sequence $\left(f^{(n)}(x)\right)_{n \geq 0}$ does not contain 1 , then it eventually becomes the cycle $(27,152)$.

Proof. We first show that

$$
f(x)<x \quad \text { for all } x \geq 1467 .
$$

Let $x \in[1467,9999] \cap \mathbb{N}$. Then, $x=(a b c d)_{10}$ for some $a, b, c, d \in\{0,1,2, \ldots, 9\}$ and $a \neq 0$. Therefore, $f(x)=S_{3,10}\left(S_{2,10}(x)\right)=S_{3,10}\left(a^{2}+b^{2}+c^{2}+d^{2}\right)$. Since $a^{2}+b^{2}+c^{2}+d^{2} \leq 4 \cdot 9^{2}=324$, we see that $f(x) \leq \max \left\{S_{3,10}(x) \mid 1 \leq x \leq 324\right\}=S_{3,10}(299)=2^{3}+9^{3}+9^{3}=1466<x$. Next suppose that $x \geq 10^{4}$ and write $x=\left(a_{k} a_{k-1} \ldots a_{1}\right)_{10}$ with $a_{k} \neq 0$. So $k \geq 5$ and $f(x)=$ 
$S_{3,10}\left(a_{k}^{2}+a_{k-1}^{2}+\cdots+a_{1}^{2}\right)$. We have $a_{k}^{2}+a_{k-1}^{2}+\cdots+a_{1}^{2} \leq 9^{2}+9^{2}+\cdots+9^{2}=81 k$ and it is easy to prove by induction on $k$ that $81 k<10^{k-1}$ for $k \geq 5$. Therefore,

$$
f(x) \leq \max \left\{S_{3,10}(x) \mid 1 \leq x<10^{k-1}\right\}=S_{3,10}(\underbrace{99 \ldots 9}_{k-1 \text { digits }})=9^{3}+9^{3}+\cdots+9^{3}=729(k-1) .
$$

It is also easy to prove by induction that $729(k-1)<10^{k-1}$ for all $k \geq 5$. So we obtain $f(x)<10^{k-1} \leq a_{k} 10^{k-1} \leq x$, as required. Hence (8) is verified. So we only need to check, for each $x \leq 1466$, whether the sequence $\left(f^{(n)}(x)\right)_{n \geq 0}$ converges to a fixed point or becomes a cycle. This can be done using a computer. We find that for each positive integer $x \leq 1466$, the sequence $\left(f^{(n)}(x)\right)_{n \geq 0}$ converges to 1 or eventually becomes the cycle $(27,152)$.

Example 2.3. Let $f=S_{3,7} \circ S_{2,5}$. Then, for each $x \in \mathbb{N}$, the sequence $\left(f^{(n)}(x)\right)_{n \geq 0}$ contains either 1, 28 or 216. Moreover, 1 and 28 are the only fixed points of $f$ and if the sequence $\left(f^{(n)}(x)\right)_{n \geq 0}$ does not contain 1 or 28 , then it eventually enters into the cycle $(216,224)$.

Proof. In this example, the bases are different (one of them is 5 and the other is 7 ). So the calculation is slightly different from the previous example. We give two solutions to this problem. We first show that

$$
f(x)<x \quad \text { for all } x \geq 7^{4} .
$$

Let $x \in \mathbb{N}$ and $x \geq 7^{4}$. Since $x \geq 5^{4}$, we can write $x=\left(a_{k} a_{k-1} \ldots a_{1}\right)_{5}$ where $k \geq 5, a_{k} \neq 0$, and $a_{i} \in\{0,1, \ldots, 4\}$ for every $i$. Then, $f(x)=S_{3,7}\left(S_{2,5}(x)\right)=S_{3,7}\left(a_{k}^{2}+a_{k-1}^{2}+\cdots+a_{1}^{2}\right)$. We have $a_{k}^{2}+a_{k-1}^{2}+\cdots+a_{1}^{2} \leq 4^{2}+4^{2}+\cdots+4^{2}=16 k$ and it is easy to prove by induction on $k$ that $16 k<5^{k-1}<7^{k-1}$ for $k \geq 5$. Now there are two ways we can proceed.

Method 1. Since $16 k<5^{k-1} \leq a_{k} 5^{k-1} \leq x$, we see that $f(x) \leq \max \left\{S_{3,7}(y) \mid 1 \leq y<x\right\}$. Let $\ell \in \mathbb{N}$ be such that $x=\left(a_{\ell}^{\prime} a_{\ell-1}^{\prime} \ldots a_{1}^{\prime}\right)_{7}, a_{\ell}^{\prime}, a_{\ell-1}^{\prime}, \ldots, a_{1}^{\prime} \in\{0,1, \ldots, 6\}$, and $a_{\ell}^{\prime} \neq 0$. Since $x \geq 7^{4}, \ell \geq 5$ and $7^{\ell-1} \leq x<7^{\ell}$. Therefore,

$$
f(x) \leq \max \left\{S_{3,7}(y) \mid 1 \leq y<7^{\ell}\right\}=S_{3,7}\left((\underbrace{66 \ldots 6}_{\ell \text { digits }})_{7}\right)=6^{3}+6^{3}+\cdots+6^{3}=216 \ell .
$$

Here we remind the reader again that $216 \ell$ is the product of the numbers 216 and $\ell$ where $216=$ $(216)_{10}$. It is also easy to prove by induction that $216 \ell<7^{\ell-1}$ for all $\ell \geq 5$. So we obtain $f(x)<7^{\ell-1} \leq x$, as required.

Method 2. We know that $16 k<7^{k-1}$, and so

$$
f(x) \leq \max \left\{S_{3,7}(y) \mid 1 \leq y<7^{k-1}\right\}=S_{3,7}\left((\underbrace{66 \ldots 6}_{k-1 \text { digits }})_{7}\right)=216(k-1) .
$$

Since $5^{k-1} \leq a_{k} 5^{k-1} \leq x$, we obtain $k-1 \leq \frac{\log x}{\log 5}$. Therefore,

$$
f(x) \leq 216(k-1) \leq \frac{216 \log x}{\log 5}=\left(\frac{216}{\log 5}\right)\left(\frac{\log x}{x}\right) x \leq\left(\frac{216}{\log 5}\right)\left(\frac{\log 7^{4}}{7^{4}}\right) x<x,
$$

where we have used the fact that $x \geq 7^{4}$ and that the function $y \rightarrow \frac{\log y}{y}$ is decreasing on $[3, \infty)$. Hence (9) is verified. Similar to Example 2.2, the rest can be verified using a computer. 


\begin{tabular}{|c|c|l|}
\hline \hline$\underline{\boldsymbol{e}}$ & $\underline{\boldsymbol{b}}$ & Fixed points of $S_{\underline{\boldsymbol{e}}, \underline{\underline{\boldsymbol{b}}}}$ or cycles in $\left(S_{\underline{\underline{e}}, \underline{\underline{b}}}^{(n)}(x)\right)_{n \geq 1}$ \\
\hline \hline$(3,2)$ & $(10,10)$ & $1,(27,152)$ \\
\hline$(2,3)$ & $(10,10)$ & $1,(30,53)$ \\
\hline$(3,2)$ & $(7,5)$ & $1,28,(216,244)$ \\
\hline$(3,4,2)$ & $(5,6,4)$ & $1,35,(17,28)$ \\
\hline$(2,3,5)$ & $(6,5,7)$ & $1,(10,20,17),(11,41)$ \\
\hline$(2,5,4)$ & $(5,6,8)$ & $1,16,19,(4,12,5,14),(7,13,27,17)$ \\
\hline$(4,3,5,2)$ & $(4,3,5,6)$ & $1,2,(98,32)$ \\
\hline$(2,3,5,4)$ & $(8,7,5,3)$ & $1,4,75,98$ \\
\hline$(4,2,3,5)$ & $(6,5,4,3)$ & $1,641,(257,625)$ \\
\hline \hline
\end{tabular}

Table 1. Fixed points of $S_{\underline{\boldsymbol{e}}, \underline{\boldsymbol{b}}}$ or cycles in $\left(S_{\underline{\boldsymbol{e}}, \underline{\boldsymbol{b}}}^{(n)}(x)\right)_{n \geq 1}$

Comments: The origin of this problem is unclear but it appears in Guy's book [5, Chapter E34]. A list of fixed points and cycles of some $S_{\underline{e}, \underline{b}}$ is given in Table 1 . We also plan to put more data in the third author's ResearchGate account, so the interested reader can freely download it in the future.

\section{Acknowledgements}

Niphawan Phoopha receives a scholarship from DPST of IPST, Thailand. Prapanpong Pongsriiam receives financial support jointly from the Thailand Research Fund and Faculty of Science Silpakorn University, grant number RSA5980040. We would like to thank both referees for their comments and suggestions, which improved the presentation and quality of this article.

\section{References}

[1] El-Sedy, E. \& Siksek, S. (2000). On happy numbers, The Rocky Mountain Journal of Mathematics, 30, 565-570.

[2] Gilmer, J. (2011). On the density of happy numbers, posted at: http: / / arxiv.org/ $\mathrm{pdf} / 1110.3836 \mathrm{v} 3 \cdot \mathrm{pdf}$

[3] Grundman, H. G. \& Teeple, E. A. (2001). Generalized happy numbers, The Fibonacci Quarterly, 39, 462-466.

[4] Grundman, H. G. \& Teeple, E. A. (2008). Iterated sums of fifth powers of digits, The Rocky Mountain Journal of Mathematics, 38, 1139-1146.

[5] Guy, R. K. (2004). Unsolved Problems in Number Theory, Springer-Verlag, Third Edition. 
[6] Hargreaves, K. \& Siksek, S. (2010). Cycles and fixed points of happy functions, Journal of Combinatorics and Number Theory, 3, 217-229.

[7] Pan, H. (2008). On consecutive happy numbers, Journal of Number Theory, 128, 16461654.

[8] Sloane, N. J. A. The On-Line Encyclopedia of Integer Sequences, https : / / oe i s . org

[9] Styer, R. (2010). Smallest examples of strings of consecutive happy numbers, Journal of Integer Sequences, 13, Article 10.6.3.

[10] Swart, B. B., Beck, K. A., Crook, S., Turner, C. E., Grundman, H. G., Mei, M. \& Zack, L. (2017). Augmented generalized happy functions, The Rocky Mountain Journal of Mathematics, 47, 403-417.

[11] Zhou, X. \& Cai, T. (2009). On e-power b-happy numbers, The Rocky Mountain Journal of Mathematics, 39, 2073-2081. 